\title{
Weak bases affect late stages of Mayaro virus replication cycle in vertebrate cells
}

\author{
D. F. FerReira, M. P. E. SANTO*, M. A. REBello and M. C. S. REBello* \\ Instituto de Microbiologia Professor Paulo de Góes and*Instituto de Biofísica Carlos Chagas Filho, \\ Universidade Federal do Rio de Janeiro, Brazil
}

\begin{abstract}
This paper describes the effect of two weak bases (ammonium chloride and chloroquine) on the morphogenesis of Mayaro virus. When Mayaro virus-infected TC7 (monkey kidney) cells were treated with these agents it was observed that weak bases caused a significant reduction in virus yield. Also, cellular protein synthesis, which is inhibited by Mayaro virus infection, recovered to nearly normal levels. However, the synthesis of Mayaro virus proteins was affected. These phenomena were dose-dependent. The process of Mayaro virus infection in vertebrate cells is very rapid. Virus precursors are not observed in cell cytoplasm and budding through the plasma membrane seems to be the only way of virus release. Electron microscopy of cells infected with Mayaro virus and treated with weak bases revealed an accumulation of virus structures in cell cytoplasm. The study also noted an inhibition of budding through the plasma membrane and the appearance of virus particles inside intracytoplasmic vacuoles. These observations indicate an impairment at the final stages of the virus replication cycle.
\end{abstract}

\section{Introduction}

Mayaro virus is a member of the Alphavirus genus, Togaviridae family and is antigenically closely related to Semliki Forest virus [1]. Mayaro virus is an arbovirus, perpetuated in nature by its ability to infect and replicate in vertebrate and invertebrate cells. The virus was isolated for the first time in Trinidad, in 1954, and since then Mayaro virus has been reported as the cause of several epidemic outbreaks in Brazil, Bolivia and other regions, mainly at the borders of colonisation in the Amazon region [2]. Clinical manifestations of human infection have been described as a feverish illness accompanied by headache, chills, nausea, photophobia, myalgia and arthralgia. In some cases there is true arthritis that persists up to months [2]. The 12-kb genome of the alphaviruses consists of a plus sense ssRNA molecule, capped at the $5^{\prime}$ terminus and polyadenylated at the $3^{\prime}$ terminus. The $5^{\prime}$ twothirds of the genome code for the non-structural proteins. The $3^{\prime}$ one-third of the genome codes for a polyprotein which will be processed and give rise to the structural proteins, consisting of a capsid protein (of c. $34 \mathrm{kDa}$ ), a precursor of $62 \mathrm{kDa}$, which will

Received 16 Feb. 1999; revised version accepted 12 July 1999.

Corresponding author: Professor M.C.S. Rebello (e-mail: IMVIDAF@microbio.ufrj.br). undergo cleavage to produce two glycoproteins (E2 and E3), and finally another glycoprotein (E1). For most alphaviruses, only glycoproteins E1 and E2, of $c$. $50 \mathrm{kDa}$ each, are structural components of the mature virion [3].

In vertebrate cells, the process of morphogenesis takes place with assembly of virus nucleocapsids in the cell cytoplasm involving association of virus capsid protein and genomic RNA, whereas the enveloping of the nucleocapsids occurs by budding, usually through the plasma membrane of the infected cells after membrane modifications by the insertion of virus-specific glycoproteins [3-5].

Weak bases, such as ammonium chloride and chloroquine, are acidotropic agents that cross membranes of endosomes and acidic organelles in their neutral form [6-8]. Once inside these internal structures, the weak base is protonated and trapped, causing osmotic influx of water to the cellular structure. The osmotic swelling is limited by a small back diffusion of the protonated base, raising the $\mathrm{pH}$ of the acidic structure [9-12]. The de-acidification of organelles such as the Golgi body inhibits the intracellular transport of proteins to the plasma membrane or other destinations inside the cell [13]. The effect of these agents on the replication of enveloped viruses has been studied mainly on the initial stages of virus infection such as internalisation 
and release of virus genome from the endocytic vesicles [14]. This study analysed the effect of ammonium chloride and chloroquine on some aspects of the replication of Mayaro virus.

\section{Materials and methods}

\section{Cell culture and virus}

The study used TC7 cells, which are cloned from the CV-1 cell line derived from the kidney of a male adult African green monkey. The cells were grown in $60-\mathrm{cm}^{2}$ glass bottles at $37^{\circ} \mathrm{C}$ in Dulbecco's modified Eagles's medium supplemented with fetal calf serum (FCS) $2 \%$ and bovine serum $8 \%$. Mayaro virus was obtained from the American Type Culture Collection, Rockville, MD, USA. Virus stock was prepared from BHK-21 cells and kept at $-70^{\circ} \mathrm{C}[15]$.

\section{Virus titration}

Infectivity titrations of Mayaro virus were performed by plaque assay in Vero cells. Virus dilutions $(0.5 \mathrm{ml})$ were added to cell monolayers in $60-\mathrm{mm}$ petri dishes that had just reached confluency. After $60 \mathrm{~min}$ at $37^{\circ} \mathrm{C}$, virus inoculum was removed and the monolayers were overlaid with $4 \mathrm{ml}$ of medium 199 supplemented with FCS $10 \%$ and agarose $0.95 \%$ and were incubated further in an atmosphere of $\mathrm{CO}_{2} 5 \%$ in air at $37^{\circ} \mathrm{C}$. At $48 \mathrm{~h}$ post-infection (hpi), the monolayers were stained with neutral red $25 \mu \mathrm{g} / \mathrm{ml}$ and the virus plaques were counted.

\section{Treatment with weak bases}

Ammonium chloride and chloroquine were purchased from Sigma. Stock solutions were prepared in culture medium and added to each experiment according to the final concentration desired. To allow the establishment of infection, the weak base (ammonium chloride or chloroquine) was added after a virus adsorption period of $1 \mathrm{~h}$. The agent was then maintained in the medium continuously until harvest.

\section{Infectivity assay}

Confluent monolayers of TC7 cells growing in scintillation vials $\left(5 \times 10^{5}\right.$ cells/vial $)$ were infected with Mayaro virus at a multiplicity of infection (m.o.i.) of $1 \mathrm{pfu} /$ cell. After incubation for $1 \mathrm{~h}$ at $37^{\circ} \mathrm{C}$, the inoculum was replaced with fresh medium containing the desired concentration of the test agent. Incubation at $37^{\circ} \mathrm{C}$ continued until 20 hpi when medium was collected and the virus was titrated by plaque assay.

\section{Protein synthesis}

Confluent monolayers of TC7 cells, growing in scintillation vials, were infected with Mayaro virus at a multiplicity of infection of $5 \mathrm{pfu} / \mathrm{cell}$. After incubation for $1 \mathrm{~h}$ at $37^{\circ} \mathrm{C}$, the inoculum was replaced with fresh medium containing the desired concentration of the test agent. After $20 \mathrm{hpi}$, the medium was removed and the cells were pulse-labelled for $1 \mathrm{~h}$ with ${ }^{35} \mathrm{~S}$ methionine $50 \mu \mathrm{Ci} / \mathrm{ml}$. The cell proteins were analysed by SDS-PAGE as described by Laemmli [16]. Gel slabs were autoradiographed with Kodak X-Omat K films. Mayaro virus proteins were designated by their relative mol. wt estimated by reference to standard proteins.

\section{Electron microscopy}

Confluent monolayers of TC7 cells were infected with Mayaro virus at an m.o.i. of $5 \mathrm{pfu} /$ cell. After incubation for $1 \mathrm{~h}$ at $37^{\circ} \mathrm{C}$, the inoculum was replaced with fresh medium with or without the desired concentration of ammonium chloride or chloroquine. After 15 hpi (before a cytopathic effect could be detected), the cells were washed with $0.1 \mathrm{M}$ sodium cacodylate buffer, $\mathrm{pH} 7.3$, containing sucrose $7 \%$, fixed in glutaraldehyde $2.5 \%, 0.2 \mathrm{M}$ sucrose and $5 \mathrm{mM} \mathrm{CaCl}_{2}$ in the same buffer for $1-2 \mathrm{~h}$ at room temperature. After three rinses in $0.1 \mathrm{M}$ sodium cacodylate buffer,

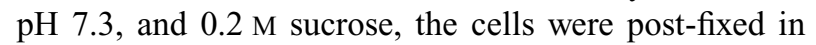
$\mathrm{OsO}_{4} 1 \% \mathrm{w} / \mathrm{v}$ in the same buffer for $1 \mathrm{~h}$ at room temperature, dehydrated in ethanol and embedded in Epon. Ultra-thin sections were stained for $20 \mathrm{~min}$ with uranyl acetate followed by $5 \mathrm{~min}$ with lead citrate and then examined in a Philips 301 transmission electron microscope operating at $80 \mathrm{kV}$.

\section{Results}

\section{Effect of weak bases on virus yield}

The study first aimed to assay the production of infectious Mayaro virus particles in TC7 cells treated with weak bases. To allow the establishment of infection, the weak base (ammonium chloride or chloroquine) was added after a virus adsorption period of $1 \mathrm{~h}$. The agent was then maintained in the medium continuously until harvest.

Table 1 shows the effect of different concentrations of ammonium chloride and chloroquine on virus yield. Both agents significantly inhibited the production of infectious Mayaro virus particles in a dose-dependent manner. The concentrations used for ammonium chloride and chloroquine apparently had no toxic effects on the cells, as revealed by trypan blue vital dye staining and by SDS-PAGE analysis of cell protein synthesis.

\section{Effect of weak bases on protein synthesis}

To determine whether the inhibition in virus yield was due to impairment of viral polypeptides expression, the TC7 cells were grown to confluence and infected with Mayaro virus $(5 \mathrm{pfu} / \mathrm{ml})$. After the adsorption period 
Table 1. Effect of weak bases on the production of infectious Mayaro virus particles

\begin{tabular}{lccc}
\hline Weak base & Concentration & Yield $(\mathrm{pfu} / \mathrm{ml})$ & Inhibition (\%) \\
\hline None (control) & - & $1.5 \times 10^{6}$ & - \\
Ammonium chloride & $5 \mathrm{mM}$ & $7.9 \times 10^{5}$ & 47.4 \\
& $10 \mathrm{mM}$ & $6.3 \times 10^{5}$ & 58.0 \\
Chloroquine & $20 \mathrm{mM}$ & $5.0 \times 10^{5}$ & 66.7 \\
& $0.1 \mathrm{mM}$ & $8.1 \times 10^{5}$ & 46.0 \\
& $0.2 \mathrm{mM}$ & $3.5 \times 10^{5}$ & 76.7 \\
& $0.3 \mathrm{mM}$ & $3.1 \times 10^{4}$ & 98.0 \\
\hline
\end{tabular}

TC7 cells were infected with Mayaro virus at an m.o.i. of 1, and after the removal of the inoculum, fresh culture medium containing either ammonium chloride or chloroquine was added. The supernates were collected at 20 hpi for virus titration. Results correspond to an average obtained from a series of three plaque assays in duplicate.

the cells were treated with different doses of ammonium chloride or chloroquine as indicated. After $20 \mathrm{hpi}$, cells were pulse-labelled with ${ }^{35} \mathrm{~S}$-methionine, lysed and the proteins were analysed by SDS-PAGE. Fig. 1a shows the protein profile of infected cells treated with increasing doses of ammonium chloride; lane 2 shows the appearance of infected untreated cells. Note the inhibition of cell protein synthesis caused by the virus infection compared with mock infected cells (lane 1), as well as the presence of the virus proteins, p34 (capsid protein), p50 (glycoprotein E2), p52 (glycoprotein E1), p62, p64 and p110, precursors of the virus proteins. When these cells were treated with ammonium chloride (lanes 3-6) a recovery of the cell protein
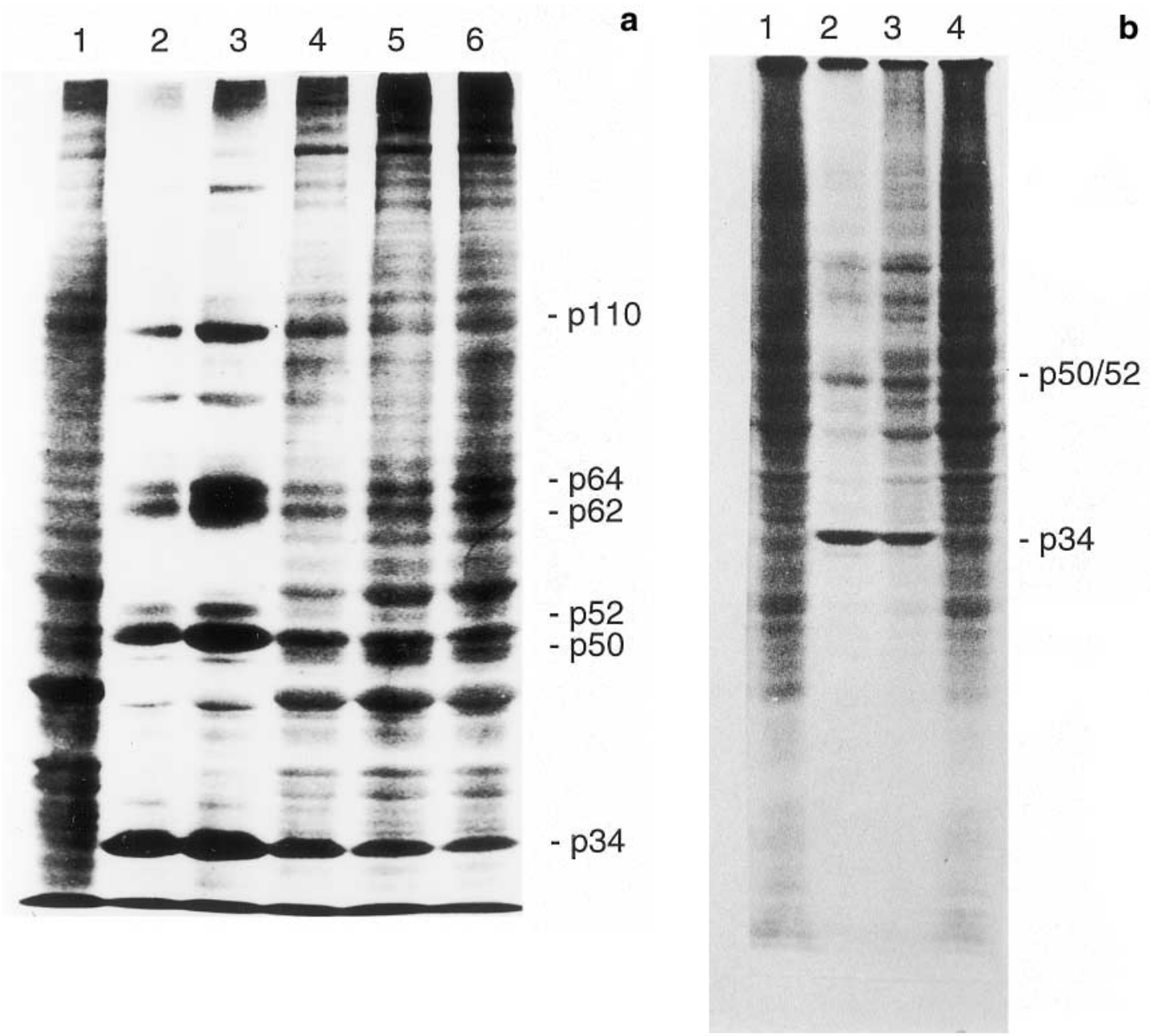

Fig. 1. The effect of weak bases on cell and virus protein synthesis. TC7 cells were infected with Mayaro virus at an m.o.i. of 5 , and after the inoculum was removed, fresh culture medium was added containing increasing doses of ammonium chloride (a) or chloroquine (b), which were kept throughout the whole experiment. At 20 hpi, the cells were pulse-labelled with ${ }^{35} \mathrm{~S}$-methionine and the proteins were analysed by SDS-PAGE . (a) Lane 1, mock infected cells; 2 , cells infected but not treated; 3-6, cells infected and treated with ammonium chloride at $10 \mathrm{~mm}, 20 \mathrm{~mm}, 30 \mathrm{mM}$ and $40 \mathrm{~mm}$ respectively. (b) Lane 1, mock infected cells; 2, cells infected but not treated; 3 and 4, cells infected and treated with chloroquine 0.1 and $0.3 \mathrm{~mm}$. 
synthesis was observed, which increased progressively with increasing concentration. At $40 \mathrm{~mm}$ ammonium chloride the cell protein profile was very similar to that of the mock infected cells (lanes 6 and 1, respectively). Virus protein synthesis was inhibited in a dosedependent manner. Similar results were found with chloroquine (Fig. 1b). Cell protein synthesis was similar to control levels with $0.3 \mathrm{mM}$ chloroquine.

\section{Effect of weak bases on morphogenesis}

Mayaro virus-infected TC7 cells were treated with ammonium chloride or chloroquine as described above. At $15 \mathrm{hpi}$, before any cytopathic effect could be detected by light microscopy, the cells were harvested and processed for electron microscopy. The results obtained with both agents were very similar. Fig. 2 shows the appearance of control (Fig. 2a) and infected (Fig. 2b, c) TC7 cells. The typical appearance of Mayaro virus infection was seen in these cells, as described by Rebello et al. [17]. A number of virus particles budding from the plasma membrane was evident in the infected cells and the study did not detect the presence of virus structures or virus precursors in the cytoplasm. Fig. 3 shows the appearance of virus-infected cells which had been treated with weak bases. Smooth vacuoles containing virus particles can be seen in the cytoplasm of infected cells (Fig. 3a and d, arrows). These particles are apparently enveloped, suggesting that many virions have formed and matured, but remain in the interior of such vacuoles. The accumulation of structures, possibly of virus origin, was also detected (Fig. 3d and e, arrowhead). These findings suggest an alteration in virus morphogenesis.

The release of virus particles from treated cells was also altered. Normal budding through the plasma membrane was rarely seen but, instead, release of virus was frequently observed in a para-crystalline fashion (Fig. 3b and c). Such a mode of release is not commonly seen in Mayaro virus-infected vertebrate cells, where budding through the plasma membrane seems to be the only way of virus release (compare with Fig. 2b and c). Apparently, the intracytoplasmic vacuoles are transported to the cell surface, where they fuse with the plasma membrane and release the particles to the extracellular environment.

\section{Discussion}

This report describes the effect of weak bases on Mayaro virus morphogenesis. First, the effect of these drugs on other stages of the virus replication cycle was analysed.

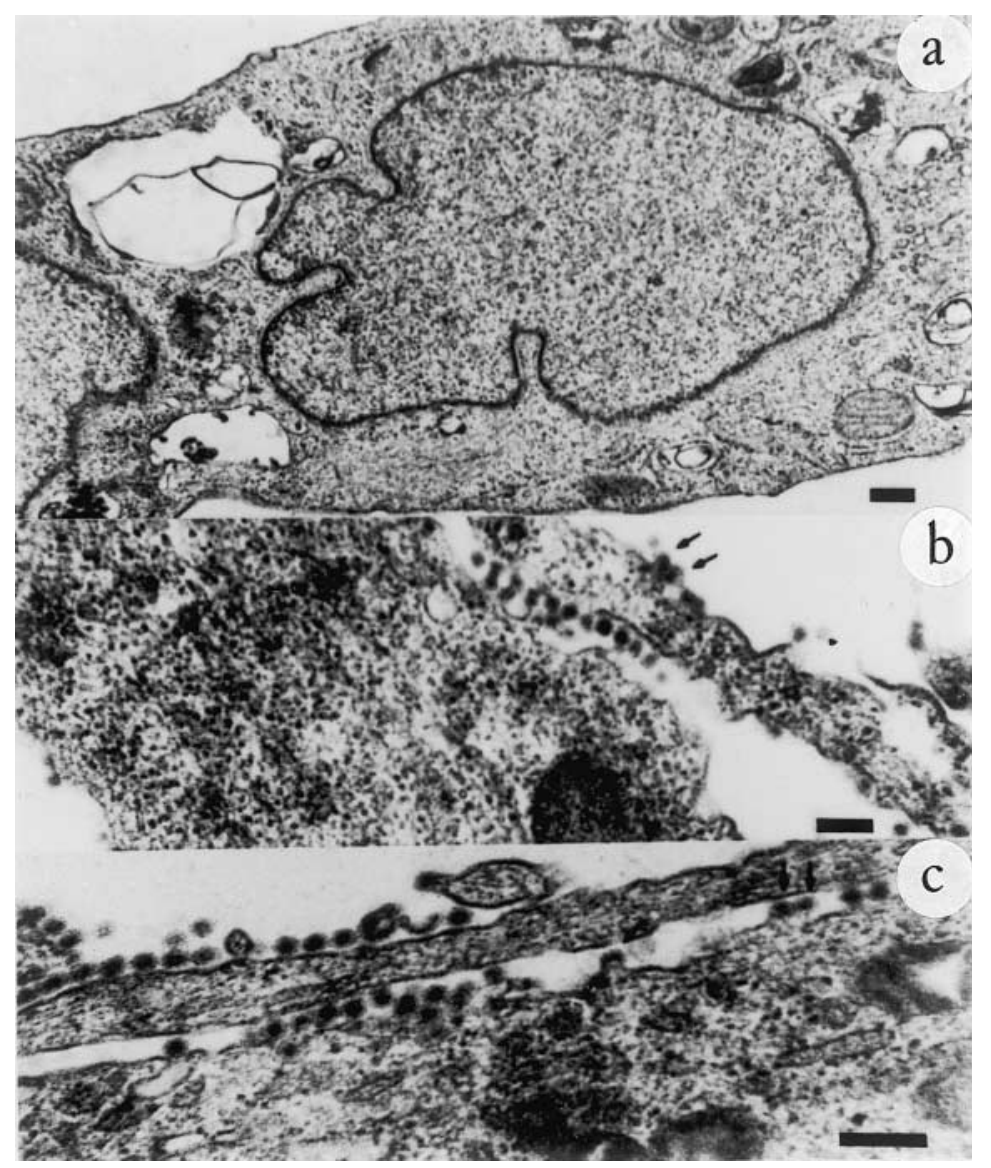

Fig. 2. Electron microscopy of TC7 cells. (a) Control uninfected cells; (b and c), untreated cells infected with Mayaro virus (m.o.i. of 5). Arrows, virus particles budding through plasma membrane. Bars, $200 \mathrm{~nm}$. 


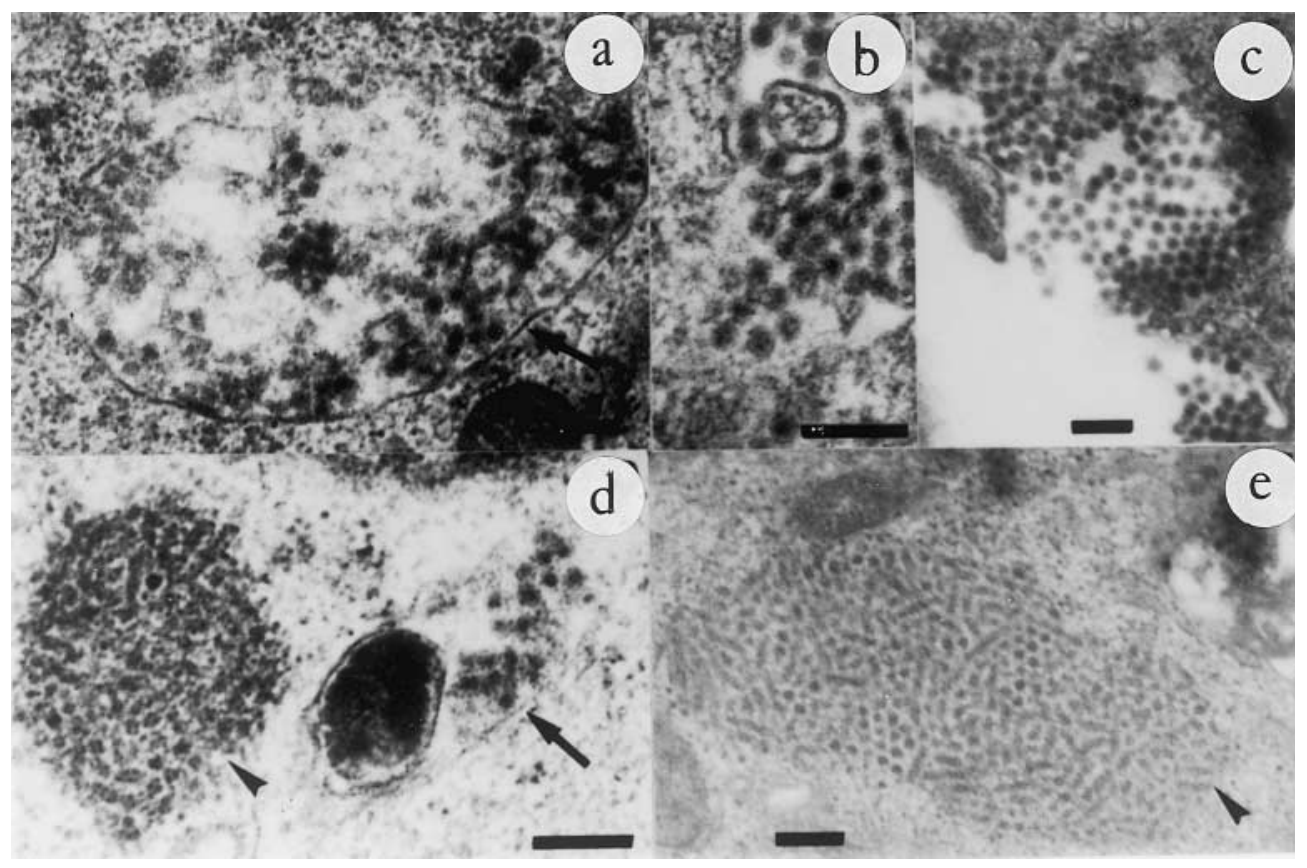

Fig. 3. Electron microscopy of TC7 cells treated with weak bases and infected with Mayaro virus. Cells were infected with Mayaro virus at an m.o.i. of 5 and treated with $10 \mathrm{mM}$ ammonium chloride (a, b) or $0.2 \mathrm{~mm}$ chloroquine (c, d, e) after removal of inoculum. At $15 \mathrm{hpi}$, cells were processed for electron microscopy. Arrows, vacuoles containing virus particles; arrowhead, accumulation of virus structures in the cell cytoplasm. Bars, $200 \mathrm{~nm}$.

In the system employed, weak bases inhibited the production of Mayaro virus infectious particles. This inhibition could be partially explained by the inhibition of virus protein synthesis in infected cells treated with weak bases. When the inhibition of virus protein synthesis was evident, it was accompanied by a restoration of the host cell protein synthesis. Several mechanisms have been proposed for the inhibition of host cell protein synthesis by alphaviruses. Frolov and Schlesinger [18] established that, in BHK cells, the synthesis of virus-specific genomic RNA or the synthesis of the non-structural viral proteins, or both, is sufficient to inhibit cell protein synthesis. It has also been suggested that the alphavirus capsid protein is involved in the shut-off of host protein synthesis [19]. Other mechanisms proposed are alterations in the ionic environment of the cell affecting the synthesis of host mRNAs more than viral mRNAs and competition between viral and host RNA [20,21]. The recovery of cell protein synthesis in the present study was proportional to the inhibition of viral protein synthesis, and perhaps the reduction in the $\mathrm{C}$ protein could also be associated with this phenomenon in the study system. However, more conclusive experiments should be performed to elucidate the mechanism(s) involved.

As has been described by others, the process of alphavirus assembly in vertebrate cells seems to be extremely rapid and efficient. Generally, viral particles free in the cytoplasm are not seen; only mature particles budding from plasma membrane have been observed [22]. These results are in agreement with the findings for TC7 cells infected with Mayaro virus
(Fig. $2 \mathrm{~b}$ and c). Mayaro virus morphogenesis is strongly affected in cells treated with weak bases. A great number of virus particles inside vacuoles and virus precursors scattered in cell cytoplasm were observed. The accumulation of virus proteins which was observed in the weak base-treated cells (Fig. 3d and e), probably virus capsid proteins and nucleocapsids, is a consequence of an impairment at late stages of the replication cycle, resulting in such accumulation of virus structures. The release of virus particles by budding through the plasma membrane is rarely seen. It appears that the release of virions from these treated cells is produced mostly by the fusion of viruscontaining vesicles with the plasma membrane.

Dille and Johnson [9] found that when vesicular stomatitis virus (VSV)-infected neuroblastoma cells were treated with chloroquine, the virus glycoproteins reached the Golgi complex but were not transported to the plasma membrane. The observations of Mayaro virus particles inside vacuoles and rare budding processes at the plasma membrane suggest that the same mechanism of inhibition of glycoprotein transport could be operating in the present study. The nucleocapsids apparently find an alternative way of completing their morphogenesis, performing the final assembly in these virus glycoprotein-bearing vacuoles. Such vacuoles are able to reach the plasma membrane and release virus particles, forming the para-crystalline release of virions observed in the present study (Fig. $3 \mathrm{~b}$ and c).

It is known that weak bases interrupt recycling of receptors and inhibit the intracellular transport of 
proteins to the plasma membrane by raising the $\mathrm{pH}$ of acidic Golgi bodies. Singh et al. [23] showed that interferon caused alkalinisation of the trans-Golgi network (TGN) and blocked the transport of herpes simplex virus-1 (HSV-1) gD glycoprotein in the TGN, a process enhanced by chloroquine. Koyama and Uchida [14], also described inhibition of the multiplication of HSV-1 at a late stage of virus infection by ammonium chloride.

The process of maturation of glycoproteins through the secretory pathway is accomplished by enzymes that require a specific $\mathrm{pH}$ to function properly. Substances such as weak bases, which alter the $\mathrm{pH}$ of these acidic organelles, may impair the function of such processing enzymes. When a virus glycoprotein is not properly processed, several aspects of the interactions of these proteins with other elements will also be affected. These include virus budding and infectivity.

In the present study, the treatment of Mayaro virusinfected vertebrate cells with drugs that inhibit virus morphogenesis resulted in an alteration of morphogenesis that very much resembled that seen for infected non-treated invertebrate cells. This has been demonstrated for weak bases, monensin (unpublished observations) and interferon [17]. It appears that treatment of vertebrate cells with these drugs forces virus morphogenesis to follow another route, i.e., intracellular virus maturation, as seen for invertebrate cells. This issue has been addressed in our laboratory, and experiments are in progress to elucidate the significance of this observation and its correlation with persistent infection.

This work was supported by Conselho Nacional de Desenvolvimento Científico e Tecnológico (CNPq) and Financiadora de Estudos e Projetos (FINEP).

\section{References}

1. Casals J, Whitman L. Mayaro virus: a new human disease agent. I. Relationship to other arbor viruses. Am J Trop Med Hyg 1957; 6: 1004-1011.

2. Causey OR, Maroja OM. Mayaro virus: a new human disease agent. III. Investigation of an epidemic of acute febrile illness on the River Guamá in Pará, Brazil, and isolation of Mayaro virus as a causative agent. Am J Trop Med Hyg 1957; 6: $1017-1023$

3. Strauss JH, Strauss EG. The alphaviruses: gene expression, replication and evolution. Microbiol Rev 1994; 58: 491-562.

4. Lopez S, Yao J-S, Kuhn RJ, Strauss EG, Strauss JH Nucleocapsid-glycoprotein interactions required for assembly of alphaviruses. J Virol 1994; 68: 1316-1323.

5. Brown DT, Waite MRF, Pfefferkorn ER. Morphology and morphogenesis of Sindbis virus as seen with freeze-etching techniques. J Virol 1972; 10: 524-536.

6. Ohkuma S, Poole B. Fluorescence probe measurement of the intralysosomal $\mathrm{pH}$ in living cells and the perturbation of $\mathrm{pH}$ by various agents. Proc Natl Acad Sci USA 1978; 75: 3327-3331.

7. Cassell S, Edwards J, Brown DT. Effects of lysosomotropic weak bases on infection of BHK-21 cells by Sindbis virus. J Virol 1984; 52: 857-864.

8. Miller D K, Lenard J. Antihistaminics, local anesthesics, and other amines as antiviral agents. Proc Natl Acad Sci USA 1981; 78: 3605-3609.

9. Dille B J, Johnson T C. Inhibition of vesicular stomatitis virus glycoprotein expression by chloroquine. J Gen Virol 1982; 62: 91-103.

10. Maheshwari RK, Sidhu GS, Bhartiya D, Friedman RM. Primary amines enhance the antiviral activity of interferon against a membrane virus: role of intracellular $\mathrm{pH}$. J Gen Virol 1991; 72: 2143-2152.

11. Koff WC, Knight V. Inhibition of influenza virus uncoating by rimantadine hydrochloride. J Virol 1979; 31: 261-263.

12. Schlegel R, Dickson RB, Willingham MC, Pastan IH. Amantadine and dansylcadaverine inhibit vesicular stomatitis virus uptake and receptor-mediated endocytosis of $\alpha_{2}$-macroglobulin. Proc Natl Acad Sci USA 1982; 79: 2291-2295.

13. Matlin KS. Ammonium chloride slows transport of the influenza virus hemagglutinin but does not cause mis-sorting in a polarized epithelial cell line. J Biol Chem 1986; 261: 15172-15178.

14. Koyama AH, Uchida T. The effect of ammonium chloride on the multiplication of herpes simplex virus type $\mathrm{I}$ in Vero cells. Virus Res 1989; 13: 271-281.

15. Mezencio JMS, de Souza W, Fonseca MEF, Rebello MA. Ultrastructural study of Mayaro virus replication in BHK-21 cells. Arch Virol 1990; 114: 229-235.

16. Laemmli UK. Cleavage of structural proteins during the assembly of the head of bacteriophage T4. Nature 1970; 227: $680-685$.

17. Rebello MCS, Fonseca MEF, Marinho JO, Rebello MA. Interferon action on Mayaro virus replication. Acta Virol 1993; 37: 223-231.

18. Frolov I, Schlesinger S. Comparison of the effects of Sindbis virus and Sindbis virus replicons on host cell protein synthesis and cytopathogenicity in BHK cells. J Virol 1994; 68: 1721-1727.

19. Elgizoli M, Dai Y, Kempf H, Koblet H, Michel MR. Semliki Forest virus capsid protein acts as a pleiotropic regulator of host cellular protein synthesis. J Virol 1989; 63: 2921-2928.

20. Contreras A, Carrasco L. Selective inhibition of protein synthesis in virus-infected mammalian cells. J Virol 1979; 29: $114-122$.

21. Kääriäinen L, Ranki M. Inhibition of cell functions by RNAvirus infections. Annu Rev Microbiol 1984; 38: 91-109.

22. Koblet H. The "merry go round": alphaviruses between vertebrate and invertebrate cells. Adv Virus Res 1990; 38: $343-402$.

23. Singh AK, Sidhu GS, Friedman RM, Maheshwari RK. Mechanism of enhancement of the antiviral action of interferon against herpes simplex virus-1 by chloroquine. J Interferon Cytokine Res 1996; 16: 725-731. 